J. Clin. Chem. Clin. Biochem.

Vol. 17, 1979, pp. 257-267

\title{
Untersuchungen über die Verwertung von $D$-Gluconat und $D$-Glucono- $\delta$-lacton im Stoffwechsel der normalen und alloxandiabetischen Ratte
}

\author{
Von L. Tharandt, W. Hübner und S. Hollmann \\ Aus dem Institut für Physiologische Chemie, Lehrstuhl I, der Universität Düsseldorf
}

(Eingegangen am 7. August/1. Dezember 1978)

Zusammenfassung: Aus Messungen der Radioaktivität im Blut normaler und alloxandiabetischer Ratten nach oraler Zufuhr von $\left[\mathrm{U}-{ }^{14} \mathrm{C}\right]$ Gluconat bzw. [U-- ${ }^{14} \mathrm{C}$ ]Glucono- $\delta$-lacton und der $5 \mathrm{~h}$ nach der Zufuhr im Darminhalt und Kot enthaltenen Restaktivität ist zu schließen, daß das Lacton intestinal besser resorbiert wird als das Gluconat-Anion. Entsprechend dieser besseren Membranpermeation und der im Blut erreichten höheren Konzentration ist der Verteilungsraum des Lactons größer als der des Gluconates (50 bzw. $41 \%$ des Körpergewichtes) und ist nach Lactonzufuhr sowohl eine höhere Retention in den Geweben als auch ein höherer Verlust im Harn festzustellen. Auch der Einbau in Leberglykogen ist nach Lacton- höher als nach Gluconatzufuhr, und zwar besonders ausgeprägt bei diabetischen Tieren. Das durch eine Latenzperiode von $7 \mathrm{bzw} .4 \mathrm{~h}$ verursachte initiale Defizit der Oxidation von Gluconat gegenüber der des Lactons wird in den darauffolgenden $8-9 \mathrm{~h}$ völlig ausgeglichen. Der oxidative Umsatz von Gluconolacton und von Gluconat ist in diabetischen Tieren signifikant gesteigert. Die bessere Verwertung im diabetischen Stoffwechsel ist z. T. auf eine Erhöhung des im Hunger und Diabetes herabgesetzten Gewebsgehaltes der Glykolysemetabolite in der Leber zurückzuführen. Limitierender Schritt des Gluconatumsatzes ist die initiale Phosphorylierung. Möglichkeiten für einen diätetischen Einsatz von Gluconsäure in Form eines apolaren Derivates (Lacton, Ester) werden diskutiert.

Investigations on the utilization of D-gluconate and D-glucono- $\delta$-lactone in the metabolism of the normal and alloxan diabetic rat

Summary: Radioactivity was measured in the blood of normal and alloxan diabetic rats, after the oral administration of $\left[\mathrm{U}-{ }^{14} \mathrm{C}\right]$ gluconate and $\left[\mathrm{U}-{ }^{14} \mathrm{C}\right]$ glucono- $\delta$-lactone, respectively. Radioactivity was also measured in the intestinal contents and feces $5 \mathrm{~h}$ after ingestion of the radioactive materials. It was concluded that the lactone is better absorbed from the intestine than the gluconate anion. According to this enhanced membrane permeation and the higher concentration reached in blood, the space of distribution of the lactone is larger than that of gluconate ( 50 and $41 \%$ of body weight, respectively); a higher retention in tissues and a greater loss in urine was also observed after administration of the lactone. Incorporation into liver glycogen is also higher from the lactone than from gluconate after oral administration, particularly in diabetic animals. The initial deficit in the oxidation of gluconate compared to that of the lactone, caused by a lag period of 7 and $4 \mathrm{~h}$, respectively, is completely compensated during the following 8-9 h. The oxidative turnover of gluconolactone and of gluconate is significantly enhanced in diabetic animals. The better utilization in diabetic metabolism is in part explainable by a rise of glycolytic intermediates in the liver, which are decreased in starvation and diabetes. The limiting step of gluconate metabolism is the initial phosphorylation. Possibilities are discussed for the dietetic use of gluconic acid in the form of an apolar derivative (lactone, ester).

\section{Einführung}

Aus dem Stoffwechselverhalten der bisher als „,Austauschkohlenhydrate" für die Diabetikerdiät empfohlenen Kohlenhydrate Sorbit (1), Fructose $(1,2)$ und Xylit $(3,4)$ ist zu schließen, daß ein Kohlenhydrat oder Kohlenhydratderivat dann für einen derartigen Austausch in Betracht kommt, wenn vor allem die folgen- den Voraussetzungen gegeben sind: Der Eintritt in die Zelle muß unabhängig von Insulin sein; das betreffende Kohlenhydrạt soll in möglichst wenigen Reaktionsschritten, die die insulinabhängige Glucokinasereaktion (5) umgehen, Anschluß an einen der Hauptwege des Kohlenhydratabbaues gewinnen und es soll den Blutglucosegehalt beim Diabetiker nach Möglichkeit nicht erhöhen. 
Diese Voraussetzungen könnte $D$-Gluconat erfüllen, das nach Phosphorylierung an C-6 (6) oder nach Dehydrierung und Decarboxylierung zu $D$-Ribulose (7) und deren Phosphorylierung via Pentosephosphatcyclus abgebaut werden kann. Bisher hat $D$-Gluconat als Zusatz zu Wurstwaren in der Lebensmittelindustrie und in der Therapie in Form des gut ionisierenden Calciumsalzes zur oralen und parenteralen Calciumzufuhr Verwendung gefunden.

Als Kohlenhydratsubstituent sind Gluconsäure und ihre Salze a priori wenig geeignet, da nur undissoziierte Säuren biologische Membranen frei permeieren können, Gluconsäure aber, pK 3,6-3,7 (8), bei physiologischem $\mathrm{pH}$ praktisch völlig dissoziiert vorliegt. Günstiger erscheint für diesen $Z$ weck das apolare $\delta$-Lacton, dessen spontane Hydrolyse intrazellulär durch die Gluconolactonase noch beschleunigt wird (9).

Die über das Stoffwechselverhalten von Gluconat und Gluconolacton bisher mitgeteilten Befunde bestätigen diese Annahme. Gluconat wird nach intravenöser oder intraperitonealer Zufuhr zu 53-85\% unverändert im Harn wieder ausgeschieden (10-12). Aus dieser Tatsache erklärt sich der Vorschlag, Gluconsäure als harnsäuerndes Agens therapeutisch zu verwenden (13). Nur maximal $15 \%$ werden in vivo $\mathrm{zu} \mathrm{CO} 2$ oxidiert $(10,11)$, wobei, dem skizzierten Abbauweg entsprechend, $\mathrm{C}-1$ bevorzugt als $\mathrm{CO}_{2}$ eliminiert wird (14). Von Glucono- $\delta$-lacton gehen dagegen nach oraler Zufuhr nur maximal 15\% durch renale Ausscheidung verloren (15). Im Wachstumstest an Ratten kann Gluconolacton in gewissen Grenzen Glucose ersetzen (16).

Im folgenden werden die Ergebnisse von Versuchen mitgeteilt, in denen die intestinale Resorption, der Verteilungsraum, die Oxidation zu $\mathrm{CO}_{2}$, der Einbau in Glykogen und die Ausscheidung im Harn und Kot von $D$ Gluconat und $D$-Glucono- $\delta$-lacton an normalen und alloxandiabetischen Ratten untersucht wurden. Zum Vergleich wurden dieselben Parameter für Glucose und teilweise auch für Sorbit ermittelt.

\section{Methodik}

Versuchstiere und Ablauf der Versuche

Die Versuche wurden an männlichen Ratten, Gewicht 180-250 g, Stamm B. R. 46-Wistar II (Tierzüchterei Brünger/Bokel) durchgeführt. Die Tiere erhielten Standardfutter der Firma Höveler, Immigrath, und Wàsser ad libitum.

Zur Erzeugung eines Alloxandiabetes wurde den Tieren nach einer $24 \mathrm{~h}$ Hungerperiode $1 \mathrm{E} / \mathrm{kg}$ Altinsulin und $1 \mathrm{~h}$ später $140 \mathrm{mg} / \mathrm{kg}$ Alloxan sowie am folgenden Tag nochmals $70 \mathrm{mg} / \mathrm{kg}$ Alloxan intraperitoneal injiziert. $\mathrm{Zu}$ den Versuchen wurden nur solche Tiere benutzt, die mindestens 10 Tage diabetisch waren und eine Glucosurie sowie einen Blutglucosegehalt von 16,7-27,8 mmol/1 aufwiesen. Die Testsubstanżen, Na-Gluconat, Glucono- $\delta$-lacton und gegebenenfalls Glucose und Sorbit, wurden den Versuchstieren nach 15-24 h Hungern in den bei den einzelnen Versuchen angegebenen Dosierungen in Form wäßr. Lösungen, 200-400 g/l, mit einer Schlundsonde verabfolgt. Da $\delta$-Lactone in wäßriger Lösung $\mathrm{pH}$-abhängig relativ leicht hydrolytisch aufgespalten werden (Glucono- $\delta$-lacton wird nach unseren Befunden bei Raumtemperatur in $5 \mathrm{~h}$ zu etwa $75 \%$ hydrolysiert), wurde eine partielle Stabilisierung durch Zusatz von Tris (hydroxymethyl)-aminomethan (Tris) zu den Glucono- $\delta$-lactonLösungen versucht. Als günstigste Bedingung erwies sich ein $\mathrm{Zu}$ satz von 1 Gewichtsteil Tris auf 4 Gewichtsteile Glucono- $\delta$-lacton (Anfangs-pH: 5,0; nach 5 h: Hydrolyse 48\%, pH 3,2). Weitere
Erhöhung der Tris-Menge begünstigt und beschleunigt wieder die Öffnung des Lactonringes.

$5 \mathrm{~h}$ nach Substratgabe bzw. nach insgesamt 20-29 h Hungern der Kontrolltiere wurden in Hexobarbitalnarkose (100$200 \mathrm{mg} / \mathrm{kg}$ ) Leberproben zur Aktivitätsmessung der unten genannten Enzyme und zur Bestimmung von Glykogen und Metaboliten entnommen. Die Entnahme der Leber zur Glykogenund Metabolitbestimmung erfolgte im Frierstoppverfahren. Muskelproben wirden aus den Oberschenkeladduktoren entnommen und sofort in flüssigem Stickstoff gefroren.

\section{Enzym bestimmungen}

Für die Enzymaktivitätsmessungen wurde 1 Gewichtsteil Leberprobe in 9 Volumteilen eiskalter Kaliumchloridlösung, $0,15 \mathrm{~mol} / \mathrm{l}$, enthaltenc $0,16 \mathrm{mmol} / 1 \mathrm{Kaliumhydrogencarbonat,}$ im Potter-Elvehjem-Homogenisator aufgeschlossen. Die Homogenate wurden in der Kühlzentrifuge $20 \mathrm{~min}$ bei $40000 \mathrm{~g}$ und die ersten Überstände nochmals $30 \mathrm{~min}$ bei $100000 \mathrm{~g}$ zentrifugiert. In den partikelfreien Überständen wurden die Aktivitäten der folgenden Enzyme bestimmt:

\section{6-Phosphogluconat-Dehydrogenase}

(6-Phospho- $D$-gluconat: NADP Oxidoreductase [decarboxylierend]; EC 1.1.1.44)

Gèsamtvolumen des Reaktionsansatzes $3,0 \mathrm{ml}$, enthaltend $125 \mu \mathrm{mol}$ Triethanolamin-Puffer, $\mathrm{pH} 7,6,30 \mu \mathrm{mol}$ Magnesiumchlorid, $1 \mu \mathrm{mol} \mathrm{NADP}+$, 0,1 ml Homogenat-Überstand; Start der. Reaktion durch Zusatz von $5 \mu \mathrm{mol}$ 6-Phosphogluconat.

\section{Transketolase}

(D-Sedoheptulose-7-phosphat: $D$-Glycerinaldehyd-3-phosphat Glycolaldehydtransferase; EC 2.2.1.1)

Bestimmung nach dem von de la Haba et al. (17) angegebenen Prinzip in einem Reaktionsansatz, Endvolumen 2,5 ml, folgen-

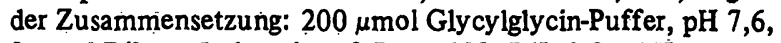
$2 \mu \mathrm{mol}$ Ribose-5-phosphat, $0,7 \mu \mathrm{mol}$ NADH, $0,2 \mathrm{ml}$ Homogenat-Überstand, $50 \mu \mathrm{l}$ Glycerin-3-phosphat: NAD 2-Oxidoreductase/ $D$-Glyceraldehyd-3-phosphat Ketol-Isomerase, EC 1.1.1.8/EC 5.3.1.1 (Boehringer Nr. 127132, 10fach verdünnt). Dieser Test basiert auf der Voraussetzung, daß die Reaktionsgeschwindigkeit durch die Aktivitäten der $D$-Ribose-5phosphat Ketol-Isomerase (EC 5.3.1.6) und der D-Ribulose-5phosphat 3-Epimerase (EC 5.1.3.1) im Homogenat nicht limitiert wird.

\section{Gluconokinase}

(ATP: D-Gluconat 6-Phosphotransferase; EC 2.7.1.12)

a) nach Ballard \& Oliver (18): Enzymatische Bestimmung des während verschieden langer Inkubation von Leberextrakt mit Gluconat + ATP gebildeten 6-Phosphogluconats,

b) im zusammengesetzten optischen Test mit 6-Phosphogluconat-Dehydrogenase als Hilfsenzym in einem Reaktionsansatz, Endvolumen 2,85 ml, der folgenden Zusammensetżung: $100 \mu \mathrm{mol}$ Triethanolamin-Puffer, $\mathrm{pH} 7,6,10 \mu \mathrm{mol}$ Na-Gluconat, $20 \mu \mathrm{mol}$ ATP, $15 \mu \mathrm{mol}$ Magnesiumchlorid, $2 \mu \mathrm{mol} \mathrm{NADP}{ }^{+}, 5 \mu \mathrm{g}$ 6-Phosphogluconat-Dehydrogenase, $0,2 \mathrm{ml}$ Homogenat-Überstand.

Mit Test a und $b$ wurden gut übereinstimmende Ergebnisse erzielt, wenn der Homogenatüberstand vor Test $b$ zwecks Entfernung freier Glucose $2-4 \mathrm{~h}$ bei $+4{ }^{\circ} \mathrm{C}$ gegen physiologische Natriumchloridlösung dialysiert wurde.

Alle Enzymaktivitäten sind in $\mathrm{U} / \mathrm{kg}$ Leberfeuchtgewicht angegeben. Meßtemperatur war stets $25^{\circ} \mathrm{C}$.

\section{Metabolitbestimmungen}

Für die Metabolitbestimmungen wurden die in flüssigem Stickstoff gefrorenen, gewogenen und pulverisierten Leberrproben mit gefrorener, pulverisierter Perchlorsäure, $0,6 \mathrm{~mol} / \mathrm{l}$, vermischt und während des Auftauens 1 min homogenisiert. Der Proteinniederschlag wurde abzentrifugiert und einmal mit $2 \mathrm{ml}$ eiskalter Perchlorsäure, $0,2 \mathrm{mò} / 1$, nachex trahiert, Dâs Geșamtextraktionsvolumen betrug stets das 8 fache der-Lebereinwaage. Nach Neutralisation der vereinigten Überstände mit Triethanolamin, 
$0,1 \mathrm{~mol} / \mathrm{l}$, enthaltend Kaliumcarbonat, $1,5 \mathrm{~mol} / 1$, wurden im klaren Überstand Glucose-6-phosphat und 6-Phosphogluconat enzymatisch bestimmt (19). Ihr Gehalt in der Leber ist angegeben als unkorrigierter Gewebsgehalt in $\mu \mathrm{mol} / \mathrm{kg}$ Feuchtgewicht.

Glykogen wurde nach Good et al. (20) aus den gefrorenen Leberund Muskelproben isoliert und als Glucose mit der Glucoseoxidase-Methode bestimmt, ebenso wie die Blutglucose. $D$ Gluconsäure- $\delta$-lacton wurde mittels der HydroxamsäureMethode (21) in der Modifikation von Eisenberg \& Field (22) bestimmt. Die photometrische Messung erfolgte konstant $1 \mathrm{~min}$ nach Eisen(III)-chlorid-Zugabe. Meßbereich 0,3-3 $\mu \mathrm{mol}$.

Alle Substrate und Enzyme für die Enzymaktivitätsmessungen und Metabolitbestimmungen wurden von der Firma Boehringer, Mannheim, Na-Gluconat, $D$-Gluconsäure- $\delta$-lacton und Sorbit von der Firma Fluka, Buchs, Schweiz, bezogen.

Versuche mit ${ }^{14} \mathrm{C}$-markierten Substraten

Je $148 \mathrm{kBq}(4 \mu \mathrm{Ci})$ der $\mathrm{U}-{ }^{14} \mathrm{C}$-markierten Substrate $D$-Glucose, Na- $D$-Gluconat bzw. $D$-Glucono- $\delta$-lacton wurden den Versuchstieren nach $15 \mathrm{~h}$ Hungern in $0,8 \mathrm{ml}$ wäßr. Lösungen, die $200 \mathrm{~g} / 1$ des betreffenden Substrates enthielten (durchschnittlich $0,8 \mathrm{~g} / \mathrm{kg}$ Körpergewicht), mittels Magensonde verabreicht. Die Lösungen des Gluconolactons waren durch Tris-Zusatz auf $\mathrm{pH} 5$ eingestellt (2,5 g Tris/10 g Lacton). Die Abatmung von ${ }^{14} \mathrm{CO}_{2}$ wurde während $15 \mathrm{~h}$ nach Substratgabe gemessen. Der Luftdurchfluß durch die Stoffwechselkäfige (Inhalt 2 1) wurde mittels Flowmeter auf $250 \mathrm{ml} / \mathrm{min}$ eingestellt. $\mathrm{Zur} \mathrm{CO}_{2}$-Absorption dienten 2 hintereinandergeschaltete Gefäße, die je $6 \mathrm{ml}$ Ethanolamin + $3 \mathrm{ml}$ Ethylenglykolmonomethylether enthielten. Das gesamte $\mathrm{CO}_{2}$ wurde stets quantitativ im ersten $\mathrm{Gefäß}$ absorbiert. Die Absorptionsgefäße wurden stündlich gewechselt. Für die Radioaktivitätsmessungen wurden je $2 \mathrm{ml}$ der auf $10 \mathrm{ml}$ aufgefüllten Absorptionsgemische eingesetzt.

Die Radioaktivität im Harn wurde in je $0,5 \mathrm{ml}$ des während $5 \mathrm{~h}$ nach Substratgabe quantitativ gesammelten Harns bestimmt. Zur Ermittlung der innerhalb von $5 \mathrm{~h}$ nach Substratgabe im Kot ausgeschiedenen und der nach $5 \mathrm{~h}$ im Darm verbliebenen Radioaktivität wurden die Tiere nach $5 \mathrm{~h}$ getötet, der gesamte Darmtrakt (Magen bis einschließlich Rectum) wurde entnommen, eröffnet und gründlich mit Wasser ausgespült. In der resultierenden Suspension wurde der während der $5 \mathrm{~h}$ abgesetzte Kot fein verteilt. Je 0,2 ml-Aliquote der gut durchmischten Suspension wurden zur Radioaktivitätsmessung eingesetzt.

Die nach $5 \mathrm{~h}$ im Gesamtorganismus verteilte Radioaktivität wurde bestimmt, indem die Kadaver, einschließlich des ausgespülten Magen-Darms, $7 \mathrm{~d}$ in verschlossenen Gefäßen bei $70^{\circ} \mathrm{C}$ in je $500 \mathrm{ml}$ Kaliumhydroxid, $300 \mathrm{~g} / 1$, inkubiert wurden. Je $5 \mathrm{ml}$ der Hydrolyselösungen wurden nach Neutralisation auf $10 \mathrm{ml}$ aufgefüllt, yon denen für die Radioaktivitätsmessung je $0,2 \mathrm{ml}$ entnommen wurden.

Für die Radioaktivitätsmessung im Blut wurde verschiedene Zeitẹn nach Substrạtzufuhr je $0,1 \mathrm{ml}$ Blut aus der Schwanzvene entnommen, mit $0,5 \mathrm{ml}$ physiologischer Natriumchloridlösung verdünnt und $10 \mathrm{~min}$ bei $3000 \mathrm{U} / \mathrm{min}$ zentrifugiert. Messung der Radioaktivität in je $0,2 \mathrm{ml}$ des klaren Überstandes. Der Umrechnung der gemessenen Aktivität auf Gesamtblutvolumen wurde der für Ratten für das gesamte Blutvolumen angegebene Wert von 6,7\% des Körpergewichtes (in g) zugrundegelẹgt (23).

Aus den 2, 5 und $10 \mathrm{~min}$ nach intravenöser Injektion von $\left[\mathrm{U}-{ }^{14} \mathrm{C}\right]$-Substraten gemessenen Radioaktivität im Blut wurde int Verteilungsraum bestimmt und in \% des Körpergewichtes angegeben. In diesen Versuchen wurde in Hexobarbitalnarkose nach Venaesectio eine mit einem $Z$ weiwegehahn verschlossene Kanüle in die Vena jugularis ext. dextra eingefürt, durch die die Substrate injiziert und die Blutpioben entnommen wurden. Nach Substratinjektion (je $24,6 \mathrm{kBq}(0,6655 \mu \mathrm{Ci})$ in $0,8 \mathrm{ml}$ einer wäßr. Lösung, $200 \mathrm{~g} / \mathrm{l}$ ) würde die Kanüle mit $0,2 \mathrm{ml}$ heparinisierter Natriumchloridlösung, $9 \mathrm{~g} / 1$, nachgespült.

Der ${ }^{14} \mathrm{C}$-Einbau in Leberglykogen wurde nach Isolierung des Glykogens zur Glykogenbestimmung (s. o.) in $0,2 \mathrm{ml}$ Proben der nach Hydrolyse und Neutralisation auf $10 \mathrm{ml}$ aufgefuillten Ânalyseniösingen bestimmt.
Alle Radioaktivitätsmessungen wurden unter $Z$ usatz von je $1 \mathrm{ml}$ Hyamin (Firma Röhm \& Haas) - nur die $\mathrm{CO}_{2}$-Absorptionslösungen erhielten keinen Hyamin-Zusatz - mit Diotol (24) als Szintillationsgemisch im Flüssigkeits-SzintillationsSpektrometer Tri-Carb (Firma Packard) durchgeführt. Quenchkorrektur erfolgte mittels externen Standards.

$\left[\mathrm{U}-{ }^{14} \mathrm{C}\right]-D$-Glucose und $\left[\mathrm{U}-{ }^{14} \mathrm{C}\right] \mathrm{Na}-D$-Gluconat (spezif. Aktivität $85 \mathrm{GBg} / \mathrm{mol}(2,3 \mathrm{mCi} / \mathrm{mmol})$ ) wurden von der Firma Buchler bezogen. $\left[\mathrm{U}-{ }^{14} \mathrm{C}\right]-D-$ Glucono- $\delta$-lacton wurde aus $\left[\mathrm{U}-{ }^{14} \mathrm{C}\right] \mathrm{Na} a-D$. Gluconat nach Umsetzung in die freie Säure mit Amberlite IR 120 und Lyophilisieren nach Isbell (25) dargestellt. Das aus Ethylenglykolmonomethylether im Stickstoffstrom auskristallisierende Präparat bestand aufgrund des Ergebnisses des Hydroxamsäure-Testes (22) zu 72,5 Tln. aus Lacton und 27,5 Tln. aus freier Säure. Eine weitere Spezifizierung war durch dünnschichtchromatographische Trennung nach

Nemec et al. (26) und Messung der Radioaktivität der anhand von Leitchromatogrammen fraktioniert abgetragenen Sorptionsschichten im Tri-Carb möglich. Nach diesen Messungen betrug das Verhältnis freie Gluconsäure: $\delta$-Lacton: $\gamma$-Lacton in unserer Präparation 28,2:61,7:10,1.

\section{Ergebnisse und Diskussion}

In ersten orientierenden Versuchen wurde unter bewußt hoher Substratdosierung geprüft, ob sich, vor allem bei Normaltieren, durch Gluconat bzw. Glucono- $\delta$ lacton eine Wiederauffüllung der durch Hunger erschöpften Leberglykogendepots erreichen läßt. Nach den in Tabelle 1 zusammengefaßten Ergebnissen erwiesen sich bei Normaltieren in absteigender Reihenfolge Glucose, Sorbit und Gluconolacton als wirksam, Gluconat zeigte keinen Effekt. Gluconolacton war umso wirksamer, je besser die Lactonstruktur durch Tris-Zusatz stabilisiert war (vgl. Methodik, Abschnitt 1). Durch intraperitoneale statt oraler Zufuhr der stabilisierten Lactonlösung in gleicher Dosierung ließ sich der Glykogengehalt bei den Normaltieren nicht weiter erhöhen ( $\bar{x} \pm \mathrm{s}: 9,9 \pm 5,1 \mathrm{~g} / \mathrm{kg}$; $\mathrm{n}=8$ ), so daß die intestinale Lactonresorption offenbar nicht als limitierender Faktor anzunehmen ist. Prinzipiell das gleiche Ergebnis, nur - Glucose ausgenommen abgeschwächt, wurde in den vergleichsweise an alloxandiabetischen Tieren durchgefürten Versuchen erhalten. Gluconolacton wirkte jedoch nur bei Tieren mit mittelschwerem Diabetes fördernd auf die Glykogensynthese, nicht dagegen bei Tieren mit einem Blutglucosegehalt über $25 \mathrm{mmol} / \mathrm{l}$.

Der Blutglucosegehalt wird durch Gluconolacton, selbst in der von uns benutzten extrem hohen Dosierung (s. Tạb. 1), nicht erhöht. Nach 24 h Hungern von Normaltieren wurde ein Mittelwert von 4,274 \pm $0,422 \mathrm{mmol} / \mathrm{l}(\mathrm{n}=10)$ und 2 bzw. $4 \mathrm{~h}$ nach Lactonzufuhr 4,241 $\pm 0,289$ ( $n=16$ ) bzw. 4,291 $\pm 0,289 \mathrm{mmol} / \mathrm{l}$ $(n=15)$ gemessen. In Anbetracht dieses Befundes war ein Einfluß von Gluconolacton auf den Glykogengehalt peripherer Gewebe von vornherein unwahrscheinlich. Diesen Erwartungen entsprechend konnte weder bei normalen noch bei diabetischen Tieren nach $24 \mathrm{~h}$ Hungern durch Gluconolactonzufuhr ein Anstieg des Muskelglykogens erzielt werden, und zwar auch dann nicht, wenn der Glykogengehalt der Muskulatur vor der Lactongabe durch ein zusätzliches Schwimmtraining 
Tab. 1. Einfluß der oralen Zufuhr von Natriumgluconat und Glucono- $\delta$-lacton auf den Leberglykogengehalt hungernder normaler und alloxandiabetischer Ratten.



*) als Glucose angegeben

**) nach $24 \mathrm{~h}$ Hungern Applikation der Testsubstanz $4 \mathrm{~g} / \mathrm{kg} \mathrm{Körpergewicht} \mathrm{in} \mathrm{wäßriger} \mathrm{Lösung} \mathrm{(400} \mathrm{g/l)} \mathrm{durch} \mathrm{Magensonde.}$ Gleiche Menge nochmals $2 \mathrm{~h}$ später. Leberentnahme $5 \mathrm{~h}$ nach erster Substratz̃ufuhr. Leberentnahme bei den Kọntrolltierẹn nach insgesamt $29 \mathrm{~h}$ Hungern.

***) bei Kollektiv I Blutzuckergehalt zwischen 16,7-25,0, bei Kollektiv II zwischen 25,0-33,3 mmol/l. Die Zahlen in Klammern geben $n$ an.

$\left(10-25\right.$ min bei $\left.+10^{\circ} \mathrm{C}\right)$ noch weiter von durchschnittlich 4,61 auf 2,23 (Normaltiere) bzw. 1,90 g/kg Feuchtgewicht (diabetische Tiere) herabgesetzt worden war. Ebenso erwies sich Sorbit in dieser Hinsicht als unwirksam. Ein signifikanter Glykogenaufbau im Muskel war unter den von uns getesteten Substraten nur durch Glucose bei Normaltieren zu erreichen.

Die im folgenden beschriebenen Versuche unter Einsatz von $\mathrm{U} \cdot{ }^{14} \mathrm{C}$-markierten Substraten in einer bei oralen Zuckerbelastungstests üblichen Dosierung wurden ausgeführt mit dem Ziel, vergleichende Bilanzen über das metabolische Verhalten von Glucose einerseits und Gluconat und Glucono- $\delta$-lacton andererseits im gesunden und diabetischen Tier aufzustellen.

Zur Abschätzung der intestinalen Resorption wurden bestimmt: Die Radioaktivität im Blut während der ersten $5 \mathrm{~h}$ nach Substratzufuhr durch Magensonde, die renale Ausscheidung während derselben Zeit, die Restaktivität im Darm und Kot nach $5 \mathrm{~h}$ und die im Gesamtorganismus (ohne Darmtrakt) nach $5 \mathrm{~h}$ retinierte Aktivität.

Der nach oraler Gluconolacton-Gabe signifikant steiler und stärker als nach Gluconatzufuhr erfolgende Anstieg der Radioaktivität im Blut (Abb. 1) und die hoch signifikanten Unterschiede der $5 \mathrm{~h}$ nach der oralen Zufuhr im Darm und Kot gemessenen Restaktivitäten (Tab. 2) sprechen eindeutig für die postulierte bessere intestinale Resorption des Lactons. Nach Gluconatzufuhr steigt die Radioaktivität im Blut während des ganzen Beobachtungszeitraumes von $5 \mathrm{~h}$ langsam ohne signifikante Differenzen zwischen normalen und diabetischen Tieren an und scheint nach $5 \mathrm{~h}$ das Maximum noch nicht erreicht $\mathrm{zu}$ haben. Nach Gluconolactonzufuhr șinkt dagegen die Radioaktivität im Blut nach Erreichen eines Maximums langsam wieder $\mathrm{ab}$, und $\mathrm{zwar}$ bei diabetischen Tieren um $1 \mathrm{~h}$ verzögert, jedoch mit nur teilweise wenig gesicherten Unterschieden $(p=0,02)$ gegenüber den Normaltieren.

Dás Erreichen gleicher Radioaktivitäten im Blut bei Normaltieren 4-5 h nach oraler Applikation von Gluconat bzw. Gluconolacton war Anlaß, den Zustand des Lactons im Magen-Darminhalt $5 \mathrm{~h}$ nach der Zufuhr zu untersuchen. Da diese Messungen eine Hydrolyse des Lactons im Darm in Höhe von 96,4\% innerhalb von $5 \mathrm{~h}$ ergaben (Hydrolyse nach 30 min bereits 63,9\%), wurde die Versuchsdauer in allen weiteren Versuchen auf $5 \mathrm{~h} \mathrm{limi-}$ tiert.

Die in gleicher Weise mit [U- $\left.{ }^{14} \mathrm{C}\right]$ Glucose als Substrat durchgeführten Versuche sind in den Abbildungen nicht wiedergegeben, da sie lediglich als Kriterien für die Beurteilung des diabetischen Zustandes der Versuchstiere dienen sollten und die bekannten Ergebnisse zeitigen: Gipfel der Radioaktivität im Blut bei Normaltieren 30 min nach der Zufuhr mit raschem exponentiellem Abfall auf etwa $26 \%$ der Maximalaktivität innerhalb der folgenden $3 \mathrm{~h}$; bei den diabetischen Tieren Verbreiterung des Gipfels mit Maximum nach $1 \mathrm{~h}$, verzögerter und verlangsamter Abfall auf etwa $41 \%$ des Maximums innerhalb der folgenden $3 \mathrm{~h}$.

Einen ungefähren Anhalt für die relative Resorptionsgeschwindigkeit von Gluconolacton vermitteln die folgenden Daten: Nach oraler Zufuhr von $148 \mathrm{kBq}$ $(4 \mu \mathrm{Ci})\left[\mathrm{U}-{ }^{14} \mathrm{C}\right]$ Gluconolacton erreichte die Radioaktivität im Blut bei normalen bzw. diabetischen Tieren maximal nur 22 bzw. $28 \%$ der nach Gabe von $148 \mathrm{kBq}(4 \mu \mathrm{Ci})\left[\mathrm{U}-{ }^{14} \mathrm{C}\right]$ Glucose gemeșenen Aktivitäten.

Außer den Ergebnissen der Resorptionsversuche weisen die gemessenen Verteilungsräume (Tạb. 3) ebenfalls auf eine bessere Membranpermeation des Lactons im Vergleich zu der des Gluconates hin, wenn auch die Differenzen statistisch nicht gesichert sind. Ohne 


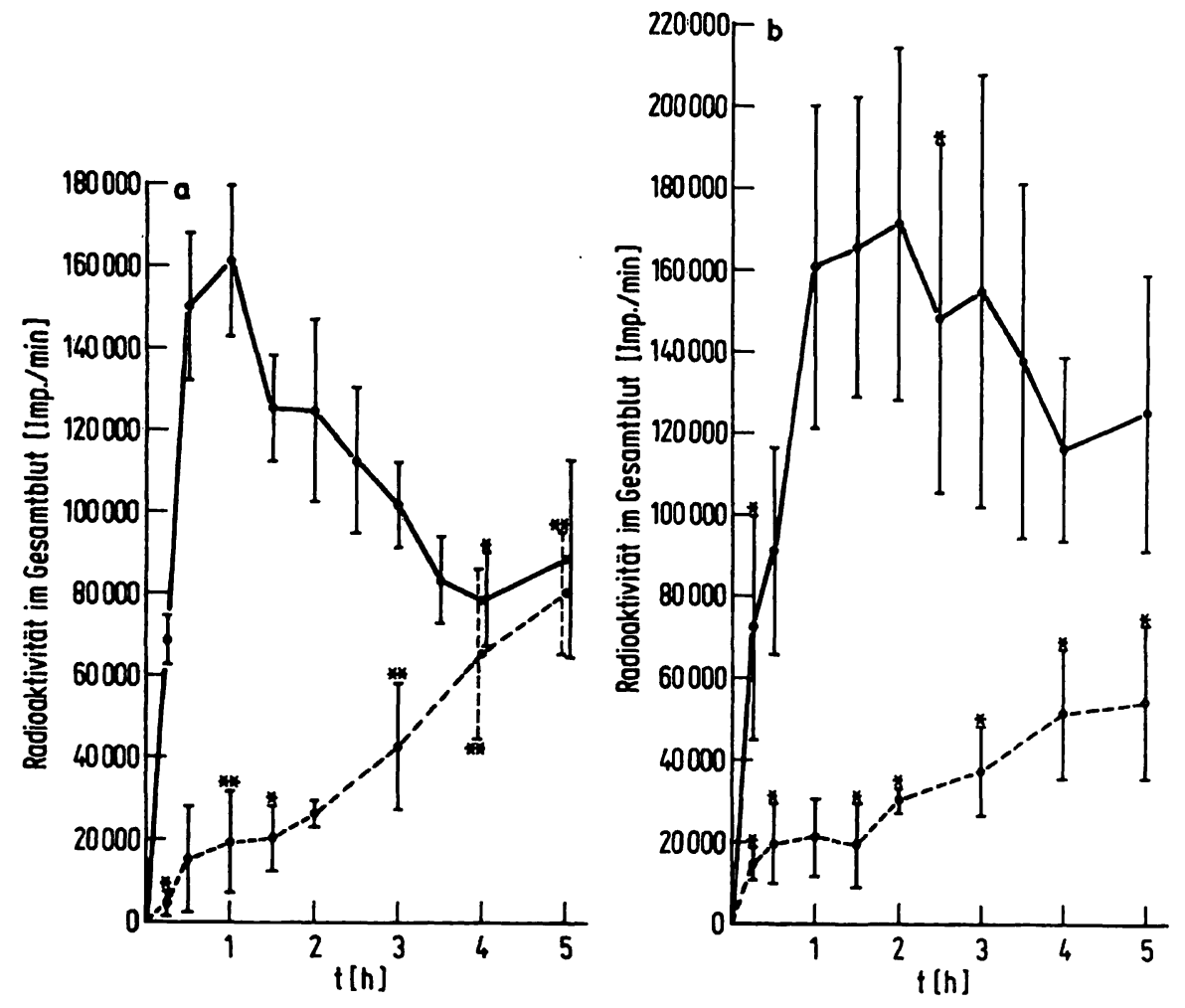

Abb. 1. Verhalten der Radioaktivität im Blut nach oraler Zufuhr von U-14 C-markiertem $D$-Gluconat bzw. $D$-Glucono- $\delta$-lacton

a) Normaltiere b) alloxandiabetische Tiere

Ordinate: Imp./min im Gesamtblut

Abszisse: h nach Zufuhr des Substrates durch Magensonde nach vorheriger $15 \mathrm{~h}$ Hungerperiode

- $148 \mathrm{kBq}(4 \mu \mathrm{Ci})\left[\mathrm{U}-{ }^{14} \mathrm{C}\right]-D-$ Glucono- $\delta$-lacton] in je $0,8 \mathrm{ml}$ wäßr. Lösungen, (,stabilisiert", s. Methodik, Abschn. 4)

- $148 \mathrm{kBq}(4 \mu \mathrm{Ci})\left[\mathrm{U}-{ }^{14} \mathrm{C}\right] \mathrm{Na}-D-\mathrm{Gluconat}$

$200 \mathrm{~g} / \mathrm{l}(\sim 0,8 \mathrm{~g} / \mathrm{kg}$ Körpergewicht)

Angegeben sind $\bar{x} \pm s$. In der Regel ist $n=5 ;$ Ausnahmen $*: n=4, * *: n=6$.

Tab. 2. Radioaktivität im Kot und Darm 5 h nach oraler Zufuhr ${ }^{14} \mathrm{C}$-markierter Substrate*).

\begin{tabular}{|c|c|c|c|}
\hline \multirow[t]{2}{*}{ Substrat } & \multicolumn{2}{|c|}{$\begin{array}{l}\text { Radioaktivität im Kot und Darm nach } 5 \mathrm{~h} \\
\text { in \% der applizierten Dosis (je } 148 \mathrm{kBq}(4 \mu \mathrm{Ci})) \overline{\mathrm{x}} \pm \mathrm{s}\end{array}$} & \multirow[t]{2}{*}{$p$ der Differenz } \\
\hline & A Normaltiere (n) & $\begin{array}{l}\text { B Alloxan- (n) } \\
\text { diabetische } \\
\text { Tiere }\end{array}$ & \\
\hline I [U-14 C]D-Glucose & $3,14 \pm 0,87(24)$ & $4,02 \pm 1,86$ & \\
\hline II [U-14 C]D-Glucono- $\delta$-lacton & $29,47 \pm 6,39(10)$ & $25,05 \pm 4,84$ & IIA/IIIA: 0,0005 \\
\hline III [U- $\left.{ }^{14} \mathrm{C}\right] \mathrm{Na}-D-\mathrm{Glu} \infty \mathrm{nat}$ & $44,86 \pm 11,56$ & $40,16 \pm 9,84(19)$ & IIB/IIIB: 0,0004 \\
\hline
\end{tabular}

*) Versuchsbedingungen s. Legende zu Abbildung 1

Tab. 3. Vertèlungsräume von $D$-Glucose, $D$-Gluconat und $D$-Glucono- $\delta$-lacton*).

\begin{tabular}{|c|c|c|c|}
\hline \multirow[t]{2}{*}{ Substrat } & \multicolumn{2}{|c|}{ Verteilungsraum in \% des Körpergewichtes $\bar{x} \pm S$} & \multirow{2}{*}{$\begin{array}{l}p \text { der } \\
\text { Differenz**) }\end{array}$} \\
\hline & $\begin{array}{l}\text { A Normaltiere } \\
\text { (n) }\end{array}$ & $\begin{array}{l}\text { B Alloxandiabetische } \\
\text { Tiere (n) }\end{array}$ & \\
\hline I [U- $\left.{ }^{14} \mathrm{C}\right] D-$-Glucose & $25,75 \pm 9,65 \quad(6)$ & $26,42 \pm 7,00(7)$ & IA/IIA: $\quad 0,013$ \\
\hline II $\left[\mathrm{U}-{ }^{14} \mathrm{C}\right] \bar{D}-$ Gluconó- $\delta$-lactón & $50,11 \pm 19,21$ & $50,43 \pm 9,64(6)$ & $\begin{array}{ll}\text { IA/IIIA: } & 0,04 \\
\text { IB/IIB: } & 0,0002\end{array}$ \\
\hline II $\left[U-{ }^{14} \mathrm{C}\right] \mathrm{Na}-D-\mathrm{Gluconat}$ & $41,40 \pm 13,04$ & $40,97 \pm 4,74$ & IB/IIIB: 0,0015 \\
\hline
\end{tabular}

*) berechnet aus der Radioaktivität im Blut 5 min nach der nach 15 h Hungern erfolgten intravenösen Injektion von je 24,6 kBq $(0,6655 \mu \mathrm{Ci})$ [U_- ${ }^{14}$ C] -Substrat in je $0,8 \mathrm{ml}$ wäßr. Lösung, $200 \mathrm{~g} / \mathrm{l}$, ohne Berücksichtigung der innerhalb der 5 min ablaufenden Oxidation und renalen Ausscheidung.

**) alle nicht aufgeführten Differenzen sind statistisch nicht signifikant. 
Unterschiede zwischen normalen und diabetischen Tieren wurde für Glucose mit durchschnittlich $26 \%$ ein Verteilungsraum gemessen, der in Ubereinstimmung mit den Ergebnissen von Baker et al. (27) kaum über dem Wert für den extrazellulären Flüssigkeitsraum liegt und signifikant niedriger ist als die für Gluconat bzw. Gluconolacton mit durchschnittlich 41 bzw. 50\% ermittelten Verteilungsräume. Gluconat und in noch stärkerem Maße Gluconolacton liegen also bereits $5 \mathrm{~min}$ nach intravenöser Injektion in beträchtlichem Umfang intrazellulär vor.

Entsprechend den nach oraler Gluconolactonzufuhr im Vergleich zu den nach Gluconatzufuhr erreichten höheren Werten der Radioaktivität im Blut (s. Abb. 1) sind nach Zufuhr des ersteren auch die Verluste im Harn geringfügig höher (Tab. 4). Hoch signifikant gesteigert sind aber die renalen ${ }^{14} \mathrm{C}$-Verluste bei diabetischen Tie- ren verglichen mit Normaltieren, und zwar stärker nach Lacton- als nach Gluconatgabe. ${ }^{14} \mathrm{C}$-Verluste in Form von Glucose sind jedoch unwahrscheinlich, da durch Gluconolacton der Blutglucosegehalt, wie gezeigt, nicht erhöht wird. Die weitgehende Parallelität zwischen Konzentration im Blut und Höhe der Ausscheidung im Harn läßt vermuten, daß beide Substanzen tubulär nicht rückresorbiert werden.

Trotz der größeren Verluste im Harn nach Gluconolacton ist der im Gesamtorganismus (ohne Darminhält) nach $5 \mathrm{~h}$ retinierte Anteil der Radioaktivität infolge der stärker gesteigerten intestinalen Resorption nach Gluconolactongabe höher àls nach Gluconatzufuhr (Tab. 5), signifikant allerdings nur bei den diabetischen Tieren.

Ein stark variierender Teil des retinierten ${ }^{14} \mathrm{C}$ ist nach $5 \mathrm{~h}$, wie aus den in Tabelle 6 zusammengefaßten Ergebnissen hervorgeht, in Leberglykogen eingebaut. Glucono-

Tab. 4. ${ }^{14} \mathrm{C}$-Ausscheidung im Harn während $5 \mathrm{~h}$ nach oraler Zufuhr ${ }^{14} \mathrm{C}$-markierter Substrate*).

\begin{tabular}{|c|c|c|c|}
\hline \multirow[t]{2}{*}{ Substrat } & \multicolumn{2}{|c|}{$\begin{array}{l}\text { Radioaktivität im Harn innerhalb von } 5 \mathrm{~h} \\
\text { nach oraler Substratzufuhr in \% der applizierten Dosis } \\
\text { (je } 148 \mathrm{kBq}(4 \mu \mathrm{Ci})) \overline{\mathrm{x}} \pm s\end{array}$} & \multirow[t]{2}{*}{$p$ der Differenz } \\
\hline & $\begin{array}{r}\text { A Normaltiére } \\
\text { (n) }\end{array}$ & $\begin{array}{l}\text { B Alloxandiabetische } \\
\text { Tiere (n) }\end{array}$ & \\
\hline I [U-14 C]D-Glucose & $1,06 \pm 0,30$ & $2,85 \pm 1,48 \quad(26)$ & \multirow{3}{*}{$\begin{array}{lc}\text { IIA/IIIA; } & 0,02 \\
\text { IIB/IIIB: } & 0,02 \\
\text { IIA/IIB: } & <0,0002 \\
\text { IIIA/IIIB: } & <0,0002\end{array}$} \\
\hline II $\left[\mathrm{U}-{ }^{14} \mathrm{C}\right] D$-Glucono- $\delta$-lacton & $7,03 \pm 1,85 \quad(10)$ & $13,82 \pm 2,83$ & \\
\hline III [U-14 C]Na-D-Gluconat & $5,03 \pm 2,16$ & $9,34 \pm 4,08 \quad(19)$ & \\
\hline
\end{tabular}

*) Versuchsbedingungen s. Legende zu Abbildung 1.

Tab. $5 .{ }^{14} \mathrm{C}$-Retention im Gesamtorganismus (ohne Darminhalt) $5 \mathrm{~h}$ nach oraler Zufuhr ${ }^{14} \mathrm{C}$-markierter Substrate*).

\begin{tabular}{|c|c|c|c|}
\hline \multirow[t]{2}{*}{ Substrat } & \multicolumn{2}{|c|}{$\begin{array}{l}\text { Radioaktivität im Gesamtorganismus } \\
\text { (ohne Inhalt des Magen-Darmtraktes) } 5 \text { h nach oraler } \\
\text { Substratzufuhr in \% der applizierten Dosis } \\
\text { (je } 148 \mathrm{kBq}(4 \mu \mathrm{Ci})) \overline{\mathrm{x}} \pm \mathrm{s}\end{array}$} & \multirow[t]{2}{*}{$p$ der Differenz } \\
\hline & $\begin{array}{l}\text { A Normaltiere } \\
\text { (n) }\end{array}$ & $\begin{array}{l}\text { B Alloxandiabetische } \\
\text { Tiere (n) }\end{array}$ & \\
\hline I [U-14C]D-Glucose & $34,74 \pm 6,91 \quad(18)$ & $20,82 \pm 5,23(10)$ & IA/IB: \\
\hline II $\left[\mathrm{U}-{ }^{14} \mathrm{C}\right] D$-Glucono- $\delta$-lacton & $23,06 \pm 2,70(10)$ & $22,81 \pm 3,12(10)$ & IIA/IIIA: 0,075 \\
\hline III [U-14 C]Na-D-Gluconat & $19,68 \pm 4,92$ & $17,54 \pm 3,82$ & IIB/IIIB: 0,008 \\
\hline
\end{tabular}

*) Versuchsbedingungen s. Legende zu Abbildung 1.

Tab. $6 .{ }^{14} \mathrm{C}$-Einbau in Gesamtleberglykogen während $5 \mathrm{~h}$ nach oraler Zufuhr ${ }^{14} \mathrm{C}$-markierter Substratẹ).

\begin{tabular}{|c|c|c|c|}
\hline \multirow[t]{2}{*}{ Substrat } & \multicolumn{2}{|c|}{$\begin{array}{l}{ }^{14} \mathrm{C} \text {-Einbau in Leberglykogen innerhalb von } 5 \mathrm{~h} \\
\text { nach oraler Substratzufuhr in \% der applizierten Dosis } \\
\text { (je } 148 \mathrm{kBq}(4 \mu \mathrm{Ci})) \overline{\mathrm{x}} \pm \mathrm{s}\end{array}$} & \multirow[t]{2}{*}{$p$ der Differenz } \\
\hline & $\begin{array}{l}\text { A Normaltiere } \\
\cdot(n)\end{array}$ & $\begin{array}{l}\text { B Alloxandiabetische } \\
\text { Tiere (n) }\end{array}$ & \\
\hline I [U-14 C $] D$-Glucose & $3,63 \pm 2,92(14)$ & $2,08 \pm 2,61 \quad(17)$ & IIA/IIIA: \\
\hline II $\left[\mathrm{U}-{ }^{14} \mathrm{C}\right] D$-Glucono- $\delta$-lacton & $0,51 \pm 0,44$ & $4,98 \pm 1,63$ & $\begin{array}{l}\text { IIB } / \text { IIIB: } \quad<0,0002 \\
\text { IIA/İIB: } \quad<0,0002\end{array}$ \\
\hline III [U-14 C]Na-D-Gluconat & $0,18 \pm 0,12(18)$ & $0,56 \pm 0,49(14)$ & $\begin{array}{ll}\text { IIA/IIIB: } & 0,004 \\
\text { IIB/IB: } & 0,0008\end{array}$ \\
\hline
\end{tabular}

*) Versuchsbedingungen s. Legende zu Abbildung 1. 
lacton erwies sich dabei als Vorstufe der Glucose des Leberglykogens bei normalen und diabetischen Tieren gegenüber Gluconat als signifikant überlegen. Noch bedeutsamer erscheint jedoch der Befund, daß im diabetischen Zustand Gluconat-C und vor allem hoch signifikant Gluconolacton-C in größerem Ausmaß in Leberglykogen eingebaut wird als im stoffwechselgesunden Tier. Der ${ }^{14} \mathrm{C}$-Einbau aus Gluconolacton in Leberglykogen der diabetischen Tiere übersteigt nicht nur den ${ }^{14} \mathrm{C}$-Einbau aus Glucose unter diabetischen Stoffwechselbedingungen, sondern erreicht sogar das gleiche Niveau wie der Einbau von Glucose in Leberglykogen der gesunden Hungertiere.

Die Messung der Substratoxidation innerhalb von $5 \mathrm{~h}$ durch Bestimmung des exhalierten ${ }^{14} \mathrm{CO}_{2}$ (Tab. 7) erbrachte prinzipiell analoge Ergebnisse wie die Versuche über den ${ }^{14} \mathrm{C}$-Einbau in Leberglykogen, jedoch mit dem Unterschied, daß in diabetischen Tieren nach Ablauf von $5 \mathrm{~h}$ zwischen Gluconolacton und Gluconat einerseits und Glucose andererseits im Ausmaß der Oxidation Differenzen nicht nachweisbar waren. Auch wurde zwischen normalen und diabetischen Tieren in der Höhe der Glucoseoxidation kein Unterschied gefunden. Nach $10 \mathrm{~h}$ bleibt in Normaltieren die Gluconatoxidation noch hinter den Oxidationsraten von Glucose und Gluconolacton zurück, in diabetischen Tieren übersteigt aber die oxidative Metabolisierung von Gluconolacton und von Gluconat die der Glucose und erreicht Werte, die signifikant über den entsprechenden Oxidationsraten der gesunden Kontrolltiere liegen.

Besser als durch diese Zahlen wird die mit unterschiedlicher Geschwindigkeit ablaufende Oxidation von Gluconat und Gluconolacton durch die Abbildung 2 verdeutlicht. Im normalen und diabetischen Stoffwechsel setzt die Oxidation von Gluconat erst nach einer Latenzperiode ein. Das hieraus gegenüber Gluconolacton in den ersten $7 \mathrm{bzw} .4 \mathrm{~h}$ resultierende Oxidationsdefizit wird in der zweiten Phase von 8-9 h Dauer ausgeglichen. Die Beschleunigung und Steigerung des oxidativen Umsatzes von Gluconolacton im diabetischen Zustand (Anhebung des Plateaus) ist signifikant $(p \leqslant 0,007)$ von der 4. bis 6. Stunde. Die bei den diabetischen Tieren noch stärker gesteigerte Oxidation des Gluconats ist an dem steileren Anstieg der Kurve mit Erhöhung und Linksverschiebung des Gipfels um 3 h zu erkennen. Die Differenzen sind von der 1 . bis zür 6. Stunde signifikant $(p<0,007)$.

Die auffällige Besseruing der Gluconatoxidation nach einer Latenzperiode ließ aufgrund der erwiesenen langen Verweildauer im Darm an die Möglichkeit denken, daß für die Phase der gesteigerten Gluconatoxidation ein intestinal entstandener bakterieller Gluconatmetabolit verantwortlich sein könnte. Diese Möglichkeit ist aber auszuschließen, da durch Sterilisierung des Darms mit einem Sulfonamid' (dreitägige Behandlung mit Sulfaguanidin (Resulfon)) die Gluconatoxidation zu keinem Zeitpunkt während der gesamten Versuchsdauer von $15 \mathrm{~h}$ beoinflußt wird. Der Umsatz von oral verabreichtem Gluconat wird also offenbar durch die intestinale Resorption limitiert.

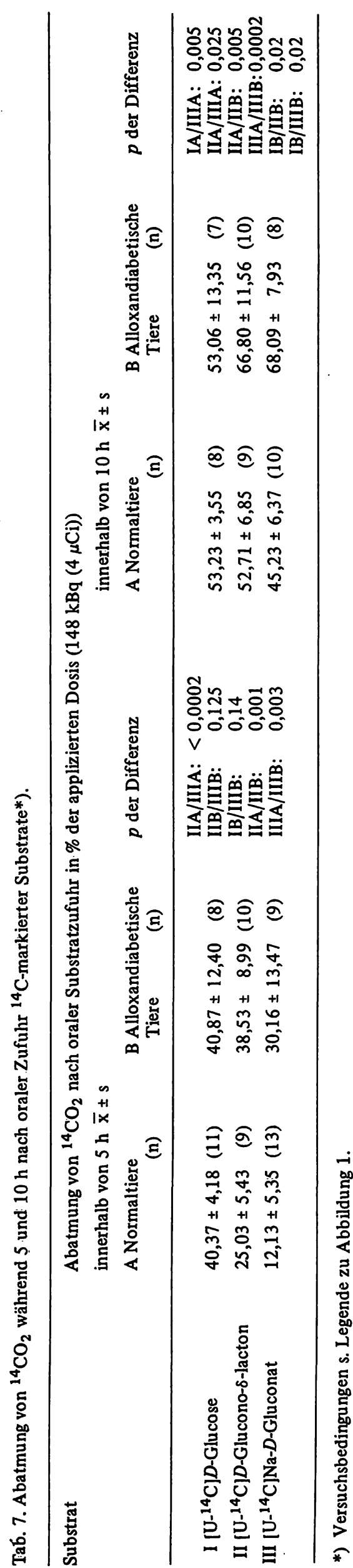

J. Clin. Chem. Clin. Biochem. / Vol. 17, 1979 / No. 4 




Abb. $2 .{ }^{14} \mathrm{CO}_{2}$-Abatmung nach oraler Zufuhr von $\mathrm{U}-{ }^{14} \mathrm{C}$-markiertem $D$-Gluconat bzw. $D$-Glucono- $\delta$-lacton*)

a) Normaltiere b) alloxandiabetische Tiere

Ordinate: Imp./min in der Ausatmungsluft pro Stunde

Abszisse: $\mathrm{h}$ nach Substratzufuhr

. $\left[\mathrm{U}-{ }^{14} \mathrm{C}\right]-D$-Glucono- $\delta$-lacton

Angegeben $\left[\mathrm{U}-{ }^{14} \mathrm{C}\right] \mathrm{Na}-D-\mathrm{Gluconat}$

Angegeben sind $\bar{x} \pm s$

n, 1.-10. h: Normaltiere: Gluconat 13; Gluconolacton 9; diabet. Tiere: Gluconat 9; Gluconolacton 10; Ausnahmen: $*: \mathrm{n}=8 ; * *: \mathrm{n}=10$

11.-15. h für alle Kollektive: $n=3$

*) Versuchsbedingungen und Substratdosierung s. Abbildung 1.

Die für den ${ }^{14} \mathrm{C}$-Einbau in Leberglykogen und die ${ }^{14} \mathrm{CO}_{2}$-Bildung innerhalb von $5 \mathrm{~h}$ nach oraler Gluconolactonzufuhir mitgeteilten Werte erlauben eine zumindest näherungsweise Schätzung der Größenordnung des Umsatzes in der Ratte. Für diabetische Tiere läßt sich aus diesen Daten ein Umsatz, bezogen auf Körpergewicht, von $\sim 390 \mu \mathrm{mol} / \mathrm{kg} \cdot \mathrm{h}$ berechnen. Zum Vergleich sei der maximale Umsatz von Xylit angeführt, der bei alloxandiabetischen Ratten mit $34-51 \mathrm{mg} \simeq 223-$ $335 \mu \mathrm{mol} / \mathrm{kg} \cdot \mathrm{h}$ bestimmt worden ist (28). Unterstellt man mit gewisser Berechtigung, daß die Metabolisierung des Gluconates im wesentlichen auf die Leber beschränkt ist, so ergibt sich aus den Gluconolacton-Versuchen in der diabetischen Rattenleber ein Umsatz von $\sim 9,8 \mathrm{mmol} / \mathrm{kg} \cdot \mathrm{h}$. Für den erwachsenen Menschen ergäben sich Umsatzraten zwischen $60-120 \mathrm{~g} /$ Tag.

Die wesentlichen vorangehend mitgeteilten Parameter des Stoffwechsels von Gluconat und Gluconolacton in normalen und diabetischen Ratten sind für die ersten $5 \mathrm{~h}$ nach Substratzufuhr in Tabelle $8 \mathrm{zu}$ einèr Bilanz zusammengefaßt. In Anbètracht der Tatsachen, daß die angegebenen Meßwerte z. T. verschiedenen Kollektiven entstammen und die individuellen Streuungen nicht in die Berechnung eingehen, sind auch die Bilanzen bei den Normaltieren mit Ausbeuten zwischen $82-85 \%$ der Theorie als durchaus befriedigend ausgeglichen anzusprechen. Nach Kenntnis des zeitlichen Verlaufes (s. Abb. 2) wären allerdings Bilanzen nach 10 oder noch besser nach $15 \mathrm{~h}$ sinnvoller gewesen.

Die bessere Verwertung von Gluconolacton und von Gluconat in diabetischen gegenüber dẹn gesunden Kontrolltieren, und żwar sowohl den Einbau in Ĝly- 
Tab. 8. Synopsis der in den Tabellen 2, 4, 5 und 7 aufgeführten Ergebnisse.

\begin{tabular}{llllr}
\hline & \multicolumn{2}{l}{ Normaltiere } & \multicolumn{3}{c}{ Diabetische Tiere } \\
& \% der verabfolgten Radioaktivität innerhalb von 5 h nach oraler Zufuhr von \\
& Na-D-Gluconat & D-Glucono- $\delta$-lacton & Na-D-Gluconat & D-Glucono- $\delta$-lacton \\
\hline abgeatmet als $\mathrm{CO}_{2}$ & 12,1 & 25,0 & 30,2 & 38,5 \\
in Geweben retiniert & 19,7 & 23,1 & 17,5 & 22,8 \\
retiniert im Darm + ausgeschieden im Kot & 44,9 & 29,5 & 40,2 & 25,1 \\
ausgeschieden im Harn & 5,0 & 7,0 & 9,3 & 13,8 \\
& & & 97,2 & 100,2 \\
Summe & 81,7 & 84,6 & & \\
\hline
\end{tabular}

Tab. 9. Gehalt der Leber normaler und alloxandiabetischer Ratten an Glucose-6-phosphat und 6-Phosphogluconat nach unterschiedlicher Vorbehandlung.

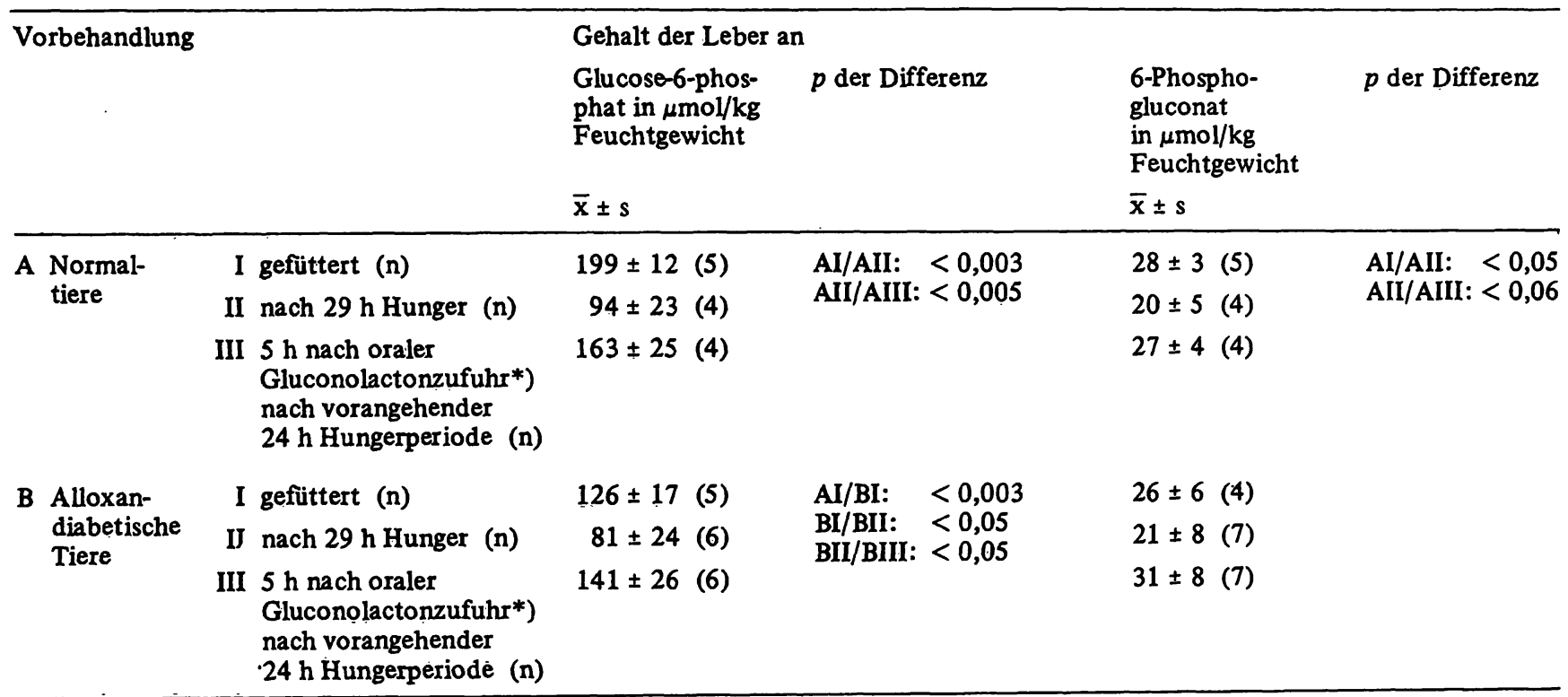

*) Nach $24 \mathrm{~h}$ Hungern D-Glucono- $\delta$-lacton, $4 \mathrm{~g} / \mathrm{kg}$ Körpergewicht in wäßr. Lösung (400 g/l) unter Zusatz von $1 / 4$ Gewichtsteil Tris durch Magen sonde. Gleiche Menge $2 \mathrm{~h}$ später nochmals. Leberentnahme $5 \mathrm{~h}$ nach der ersten Zufuhr.

kogen als auch den oxidativen Abbau betreffend, könnte dadurch zustandekommen, daß der durch Diabetes und zuusätzlich durch Hunger stark herabgesetzte Gewebsgehalt der Glykolysemetabolite in der Leber durch Zustrom von Fructose-6-phosphat und Glycerinaldehyd-3-phosphat aus dem Umsatz von $D$-Gluconat auf 2 Ebenen wieder aufgefüllt wird. Tatșächlich steigt, wie Tabelle 9 zeigt, der Gehalt an Glucōse-6-phosphat in der Leber der normalen und diabetischen Hungertiere nach Gluconolactonzufuhr an, aber in etwa gleichem Umfang bei den beiden Kollektiven und ohne das bei gefütterten Normaltieren gemessene Niveau zu errreichen. Diese erste orientierende Stichprobe nach $5 \mathrm{~h}$ erlaubt zwar noch keine eindeutige Schlußfolgerung, da die sehr wesentliche Zeitabhängigkeit nicht berücksichtigt worden ist und zudem Gewebsgehalte nichts über den Durchsatz in einer Stoffwechselkette aussagen. Offenbar reicht aber die Erhöhung der Gewebsgehalte der Glykolysemetabolite durch Gluconat bzw. Gluconolacton allein nicht aus, um die quantitativen Unter- schiede im Stoffwechsel der normalen und diabetischen Tiere befriedigend zu erklären.

Der Gewebsgehalt an 6-Phosphogluconat in der Leber, der wegen der erwiesenen Hemmwirkung von 6-Phosphogluconat auf Phosphoglucose-Isomerase $(29,30)$ und auf Phosphoribose-Isomerase (31) gemessen wurde, folgt unter allen in der Tabelle 9 angegebenen Versuchsbedingungen dem des Glucose-6-phosphates, nur auf niedrigerem Pegel. Unterschiede zwischen normalen und diabetischen Tieren wurden nicht festgestellt. Da der Gewebsgehalt an 6-Phosphogluconat in der Leber nach Gluconolactonzufuhr den der gefütterten Normaltiere nicht übersteigt, d. h. es nicht zu einem 6-Phosphogluconat-Anstau kommt, ist mit einer Isomerasehemmung unter Einfluß des Gluconolactons nicht zu rechnen.

Da seit den Arbeiten von Glock \& McLean (32) bekannt ist, daß im Hunger und im Diabetes die Aktivität der den Pentosephosphatcyclus einleitenden beiden Dehydro- 
genasen herabgesetzt ist, wurde auch der Einfluß von Hunger und Diabetes auf die Aktivität der für den Gluconatabbau erforderlichen ersten drei Enzyme untersucht. Für die 6-Phosphogluconat-Dehydrogenase konnte die Erniedrigung der Aktivität im Hunger und Diabetes, die sich bei diabetischen Hungertieren summiert, bestätigt werden. Eine signifikante Senkung der Transketolaseaktivität wurde nur bei den diabetischen Hungertieren gefunden (Normaltiere, gefüttert: 1,21, Hungertiere 1,13; diabetische Tiere, gefuittert: 1,04, Hungertiere: $0,84 \mathrm{kU} / \mathrm{kg}$ Leberfeuchtgewicht). Für die Gluconokinase wurden mit Werten zwischen 186-228 U/kg Leberfeuchtgewicht die niedrigsten Aktivitäten unter den 3 Enzymen gemessen, die zudem keine gesicherten $\mathrm{Ab}$ hängigkeiten vom Stoffwechselzustand erkennen ließen. Weder eine einmalige orale Gabe von Gluconolacton noch eine orale Behandlung diabetischer Tiere über $10 \mathrm{~d}$ mit $4 \mathrm{~g} / \mathrm{kg} \cdot \mathrm{d}$ übte auf die Aktivität der drei genannten Enzyme einen eindeutigen Einfluß aus. Wie in der Regel in Stoffwechsel-Cyclen bzw. -Ketten, scheint auch für den Gluconatumsatz die Initialreaktion die Schrittmacherreaktion zu sein; denn die Aktivität der Gluconokinase in der Leber liegt durchschnittlich eine Potenz niedriger als die der 6-Phosphogluconat-Dehydrogenase. Untersuchungen über die Regulation der Gluconokinase sind daher für das Verständnis der von uns erhobenen Befunde unerläßlich.

Die über den Stoffwechsel von Gluconat/Gluconolacton erhobenen Befunde weisen in vielen Beziehungen Ähnlichkeiten mit dem metabolischen Verhalten des Xylits auf. Wie gezeigt, erhöht Gluconolacton nicht den Blutglucosegehalt und wird Gluconolacton-C im diabetischen Tier in beachtlichem Ausmaß in Leberglykogen eingebaut. Gluconat und sein $\delta$-Lacton werden zwar langsam, aber in hohem Prozentsatz oxidativ metabolisiert, vor allem im diabetischen Zustand. Ein senkender Effekt auf die Ketonurie bei alloxandiabeti- schen Ratten ist für Gluconat (33) und auf den Gehalt an freien Fettsäuren im Serum für Gluconolacton (34) nachgewiesen worden. Auch bezüglich der ursächlich noch nicht völlig geklärten besseren Verwertung im diabetischen Organismus besteht Analogie zur Xylitverwertung in dieser Stoffwechselsituation $(4,28)$. Trotz dieser sehr günstigen Eigenschaften kommt dem Gluconat/ Gluconolacton als „Austauschkohlenhydrat“ in der Diabetes-Diätetik wohl kaum eine Bedeutung zu. Denn ebenso wie die anderen für diesen Zweck bisher empfohlenen, insulinunabhängig verwertbaren Kohlenhydrate scheint auch Gluconat im wesentlichen in der Leber, nicht aber in der Muskulatur umgesetzt zu werden. Ferner sprechen die fehlenden geschmacklichen Qualitäten gegen eine diätetische Verwendung.

Dagegen erscheint es erwägenswert, die beim Gluconatabbau erfolgende Reduktion von NADP bei den genetischen Defekten der Glucose-6-phosphat-Dehydrogenase der Erythrocyten therapeutisch zu nutzen, vorausgesetzt, daß menschliche Erythrocyten eine Gluconokinase ausreichender Aktivität enthalten. Sollte diese Voraussetzung zutreffen, so könnte durch Zufuhr von Gluconat oder eines Gluconsäure-Derivates eine Besserung der klinischen Symptomatik erzielt werden, da unter Umgehung des genetischen Defektes die Bildung von Reduktionsäquivalenten in Form von NADPH gewährleistet würde. Für den genannten therapeutischen Zweck wäre Gluconolacton wegen der rascheren Resorption und des größeren Verteilungsraumes sicher vorteilhafter als Gluconat. Aufgrund der rascheren Resorption tritt nach oraler Lactongabe $(0,8 \mathrm{~g} / \mathrm{kg})$ nur selten eine osmotisch bedingte Diarrhoe auf, während wir nach oraler Zufuhr von Gluconat in gleicher Dosis - wie nach der Zufuhr anderer langsam resorbierbarer Zucker und Polyalkohole - eine Diarrhoe bei etwa $25 \%$ unserer Versuchstiere beobachteten. Der größere Verteilungsraum würde ferner einen gößeren Substratnachschub an das Blut aus den wenig stoffwechselaktiven Geweben ermöglichen. Gegebenenfalls wäre zu erwägen, anstelle des spontan hydrolysierenden Lactons ein anderes apolares, in wäßriger Lösung stabiles GluconsäureDerivat auf seine Verwendbarkeit zu testen, etwa einen Ester, aus dem Gluconat erst intrazellulär freigesetzt wird. Für die. Verwertung eines Gluconsäureesters in Erythrocyten müßte. zusätzlich zur Gluconokinase eine unspezifische Esterase vorhanden sein.

\section{Literatur}

1. Mehnert, H., Stuhlfauth, K., Mehnert, B., Wiener, L. \& Hoeflmayr, A. (1960), Münch. Med. Wochenschr. 102, 276-279.

2. Renold, A. E. \& Thorn, G. W. (1955), Amer. J. Med. 19, 163-168.

3: Prellwitz, W. \& Bässler, K. H. (1963), Klin. Wochenschr. $41,196-199$.

4. Mehnert, H., Summa, J. D. \& Förster, H. (1964), Klin. Wochenschr. 42, 382-387.

5. Salas, M., Viñ̃uela, E. \& Sols, A. (1963), J. Biol. Chem. 238, 3535-3538.

6. Leder, I. G. (1957), J. Biol. Chem: 225, 125-136.

7. Kagawa, Y., Kameyama, T., Mano, Y.\& Shimazono, N. . (1960), Biochim. Biophys. Acta 44, 205-206.

8. Bentley, R. \& Neuberger, A. (1949), Biochem. J. 45, 584-590.

9. Brodie, A. F. \& Lipmann, F. (1955), J. Biol. Chem. 212, 677-685.

10. Stetten, M. R. \& Stetten, D., Jr. (1950), J. Biol. Chem. 187, 241-252.

11. Stetten, M. R. \& Topper, Y. J. (1953), J. Biol. Chem. 203, 653-664.
12. Crawford, M. A., Loughridge, L., Milne, M. D. \& Scribner, B. H. (1959), J. Clin. Pathol. 12, 524-529.

13. Sisk, I. R. \& Toenhart, O. (1938), J. Urol. 39, 699-709.

14. Wang, C. H., Snipper, L. P., Bilen, O. \& Hawthorne, B. (1962), Proc. Soc. Exp. Biol. Med. 111, 93-97.

15. Chenoweth, M. B., Civin, H., Salzman, C., Cohn, M. \& Gold, H. (1941), J. Lab. Clin. Med. 26, 1574-1582.

16. Eyles, R. \& Lewis, H. B. (1943), J. Nutrition 26, 309-317.

17. de la Haba, G., Leder, I. G. \& Racker, E. (1955), J. Biol. Chem. 214, 409-426.

18. Ballard, F. J. \& Oliver, I. T. (1964), Biochem. J. 92, 131136.

19. Hohorst, H. J. (1970), in Methoden der enzymatischen Analyse (Bergmeyer, H. U., Hrsg.) 2. Auflage, Bd. II, 1200-1204, 1210-1212, Verlag Chemie, Weinheim/Bergstr.

20. Good, C. A., Kramer, H. \& Somogyi, M. (1933), J. Biol. Chem. 100, 485-491.

21. Hestrin, S. (1949), J. Biol. Chem. 180, 249-261.

22. Eisenberg, F., Jr. \& Field, J. B. (1956), J. Biol. Ch̆em. 222, 293-300.

23. Hagemann, E. \& Schmidt, G. (1960), Ratte und Maus, Verlag Walter de Gruyter \& Co., Berlin.. . 
24. Herberg, R. J. (1960), Anal Chem. 32, 42-46.

25. Isbell, H. S., Karabinos, J. V., Frush, H. L., Holt, N. B., Schwebel, A. \& Galkowski, T. T. (1952), J. Res. Nat. Bur. Stand. 48, 163-171.

26. Němec, J., Kefurt, K. \& Jary, J. (1967), J. Chromatogr. 26, 116-120.

27. Baker, N., Shipley, R. A., Clark, R. E. \& Incefy, G. E. (1959), Amer. J. Physiol. 196, 245-252.

28. Schmidt, B., Fingerhut, M. \& Lang, K. (1964), Klin. Wochenschr. 42, 1073-1077.

29. Parr, C. W. (1956), Nature (London) 178, 1401.
30. Noltmann, E. \& Bruns, F. H. (1959), Biochem. Z. 331, 436-445.

31. Dobrogosz, W. J. \& Demoss, R. D. (1963), Biochim. Biophys. Acta 77, 629-638.

32. Glock, G. E. \& McLean, P. (1955), Biochem. J. 61, 390397.

33. Koster, R. (1951), Fed. Proc. Fed. Amer. Soc. Exp. Biol. $10,315-316$.

34. El Mofty, A., Khattab, M., Fayer, K. J. \& El Ridi, M. S. (1964), J. Chem. U. A. R. 7, 15-29.

Prof. Dr. S. Hollmann, Institut für Physiologische Chemie, Lehrstuhl I,

der Universität Düsseldorf

Moorenstraße 5

D-4000 Düsseldorf 



\section{Walter de Gruyter Berlin. New York}

\section{Gideon Blauer Horst Sund} (Editors)

\section{Transport by Proteins}

\section{Proceedings of a Symposium held at the University of Konstanz, West Germany, July 9-15, 1978 FEBS Symposium No. 58}

\author{
Edited by Gideon Blauer, Ph. D., Professor of Biophysical \\ Chemistry, Institute of Life Sciences, The Hebrew \\ University, Jerusalem/lsrael and Horst Sund, \\ Dr. rer. nat., Professor of Biochemistry, Biology \\ Department, University of Konstanz/West Germany.
}

$1978.17 \mathrm{~cm} \times 24 \mathrm{~cm}$. XV, 420 pages. With numerous figures and tables. Hardcover DM 145,ISBN 3110076942

This book presents the proceedings (lectures and discussions) of a Symposium on "Transport by Proteins", held at the University of Konstanz. Well-known scientists discuss general problems, selected transport proteins, membranes and transport by proteins, metal transport by proteins and the molecular aspects of transport by proteins in physiology and pharmacology.

The topic covers a wide range of different systems of fundamental physiological significance, such as the transport of oxygen, metabolites, metal ions, drugs, hormones etc., all mediated by proteins either in aequeous solution or through membraneous structures, including multi-enzyme complexes and the oxidation-reduction chains of respiration and photosynthesis. 

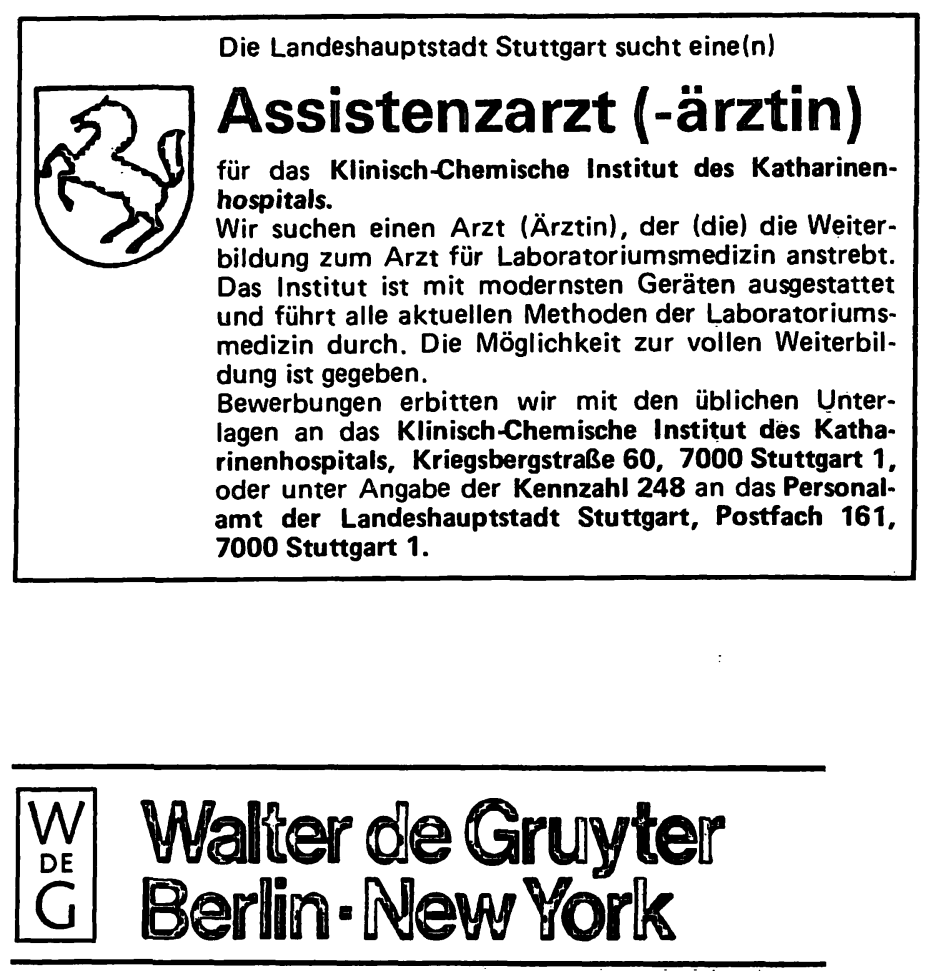

\section{Walter de Gruyter Berlin.New York}

\author{
Eckhart Buddecke
}

\section{Pathobiochemie}

\section{Ein Lehrbuch für Studierende und Ärzte}

\section{$17,0 \mathrm{~cm} \times 24,0 \mathrm{~cm}$. XXXVI, 446 Seiten.} Mit 247 Abbildungen und zahlreichen Tabellen. 1978. Plastik flexibel DM 34,ISBN 3110075261

Das Lehrbuch Pathobiochemie beschreibt die biochemischen Grundlagen genetischer und erworbener Störungen des Stoffwechsels, Abweichungen in der chemischen Struktur der Bausteine des menschlichen Körpers, fehlërhafte biochemische Prozesse, soweit sie sich als Symptome mit Krankheitswert manifestieren.

Als einführendes Lehrbuch umfaßt die Pathobiochemie die Hauptabšchnitte: Stoffwechsel, Stoffwechselregulation, Zellen, Gewebe und Organe und Dynamische Systeme.

Die Pathobiochemie liefert der Medizin neue theoretische Grundlagen, zeigt, in welchen Bereichen ein erfolgreicher Vorstoß bis zu einer "molekularen Krankheitslehre“" möglich ist, und vermittelt als fachgebietsübergreifende Wissenschaft auch Beziehungen zu zahlreichen Nachbargebieten wie z. B., zu Pathologie, Immunologie; Pharmakologie, Klinischer Chemie und Innerer Medizin.

Bei der Auswahl des Stoffes wurde die revidierte und neugegliederte 2. Auflage (1978) des Gegenstandskataloges „Pathophysiologie und Pathobiochemie" eingehend berücksichtigt.

Ein entsprechendes Korrelationsregister zum GK ist dem Buch beigefügt.

Preisãnderungen vorbehalten 